\title{
Theoretical Modeling of Electronic Structures of Polyiodide Species Included in $\alpha-$ Cyclodextrin
}

Masaki Okuda, ${ }^{1}$ Takato Hiramatsu, ${ }^{2}$ Mitsuru Yasuda, ${ }^{3}$ Mika Ishigaki, ${ }^{4,5}$ Yukihiro

Ozaki, ${ }^{1,3}$ Michitoshi Hayashi, ${ }^{6}$ Keisuke Tominaga, ${ }^{1,2}$ and Eri Chatani ${ }^{2}$

${ }^{1}$ Molecular Photoscience Research Center, Kobe University, 1-1 Rokkodai, Nada, Kobe, Hyogo 657-8501, Japan

${ }^{2}$ Department of Chemistry, Graduate School of Science, Kobe University, 1-1 Rokkodai, Nada, Kobe, Hyogo 657-8501, Japan

${ }^{3}$ School of Science and Technology, Kwansei Gakuin University, 2-1, Gakuen, Sanda, Hyogo 669-1337, Japan

${ }^{4}$ Raman Project Center for Medical and Biological Applications, Shimane University, 1060

Nishikawatsu, Matsue, Shimane 690-8504, Japan

${ }^{5}$ Faculty of Life and Environmental Sciences, Shimane University, 1060 Nishikawatsu,

Matsue, Shimane 690-8504, Japan

${ }^{6}$ Center for Condensed Matter Sciences, National Taiwan University, 1 Roosevelt Rd., Sec. 4, Taipei, 10617, Taiwan

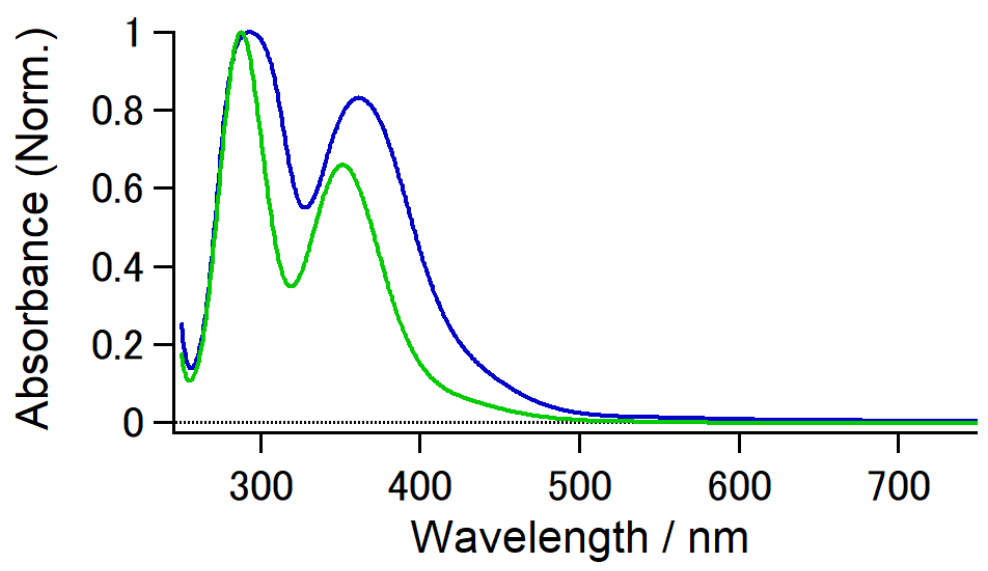

Figure S1. UV-visible absorption spectra for a supernatant solution of iodine-stained $\alpha-C D$ (blue) and iodine solution without $\alpha-\mathrm{CD}$ (green). The spectra were normalized using the maximum intensities of peaks at $\sim 300 \mathrm{~nm}$. 
(a)

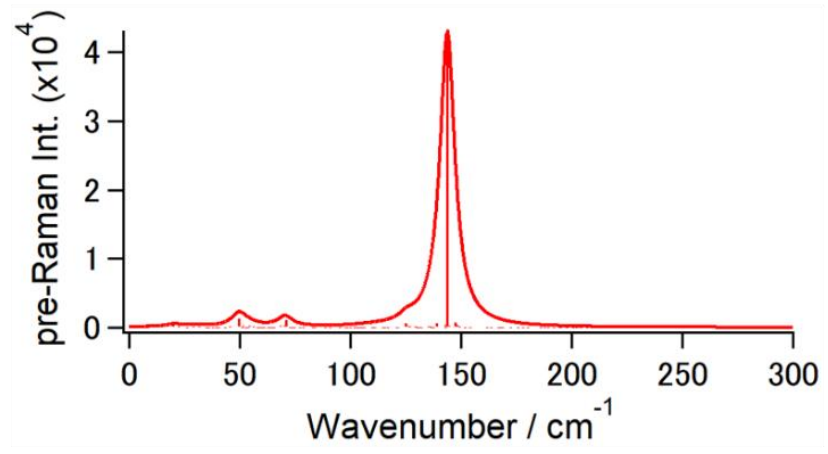

(b)

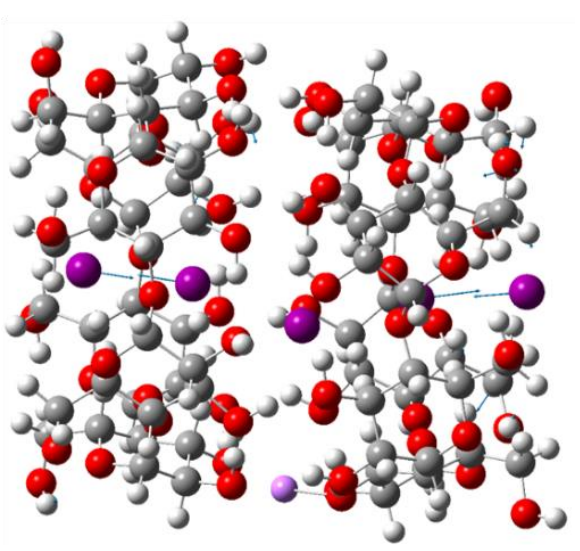

Figure S2. (a) Preresonance Raman spectrum of $\mathrm{I}_{5}{ }^{-} / \mathrm{Li}^{+} / 2 \alpha-\mathrm{CD}$ in the gas phase calculated by the CAM-B3LYP method with the LanL2DZ basis set for iodine, the LanL2DZ basis set (D95V) for lithium cations and the $6-31 * \mathrm{G}$ basis set for carbon, oxygen, and hydrogen atoms. The wavelength of incident electronic field is $514.5 \mathrm{~nm}$. The molecular structure used for this TDDFT calculation is shown in Figure 3. This Pre-resonance Raman spectrum is numerically calculated by assuming one Gaussian function with a bandwidth of $4 \mathrm{~cm}^{-1}$ for each normal mode. (b) A molecular motion of the dominant Raman active mode ( 75 th normal mode, $143.85 \mathrm{~cm}^{-1}$ ). 
(a)

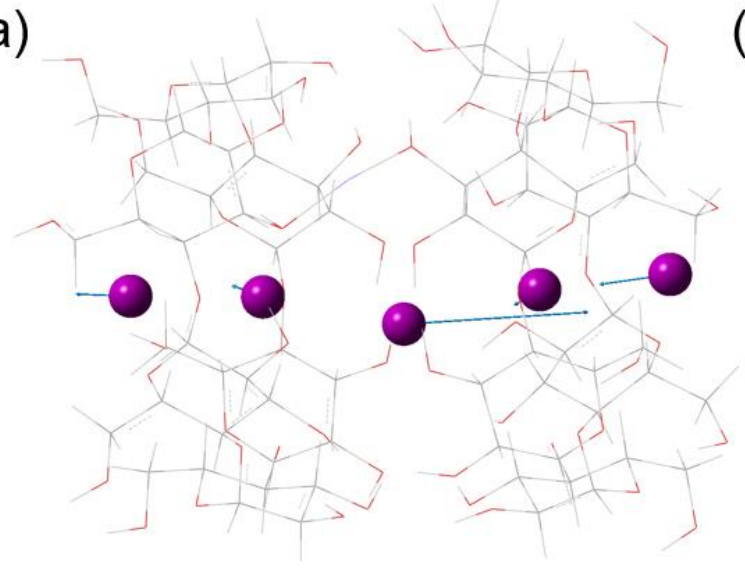

(b)

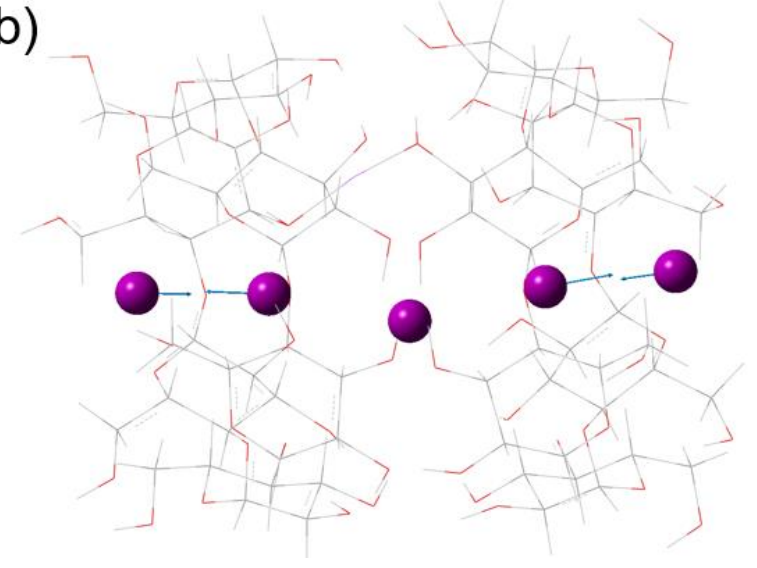

Figure S3. Molecular motions of $\mathrm{I}_{5}^{-} / \mathrm{Li}^{+} / 2 \alpha-\mathrm{CD}$ in the gas phase obtained by QM/MM calculations. The displacement vector for each atom is indicated by the blue arrow. (a) Mode 13 at $117.17 \mathrm{~cm}^{-1}$ and (b) mode 15 at $159.32 \mathrm{~cm}^{-1}$. 

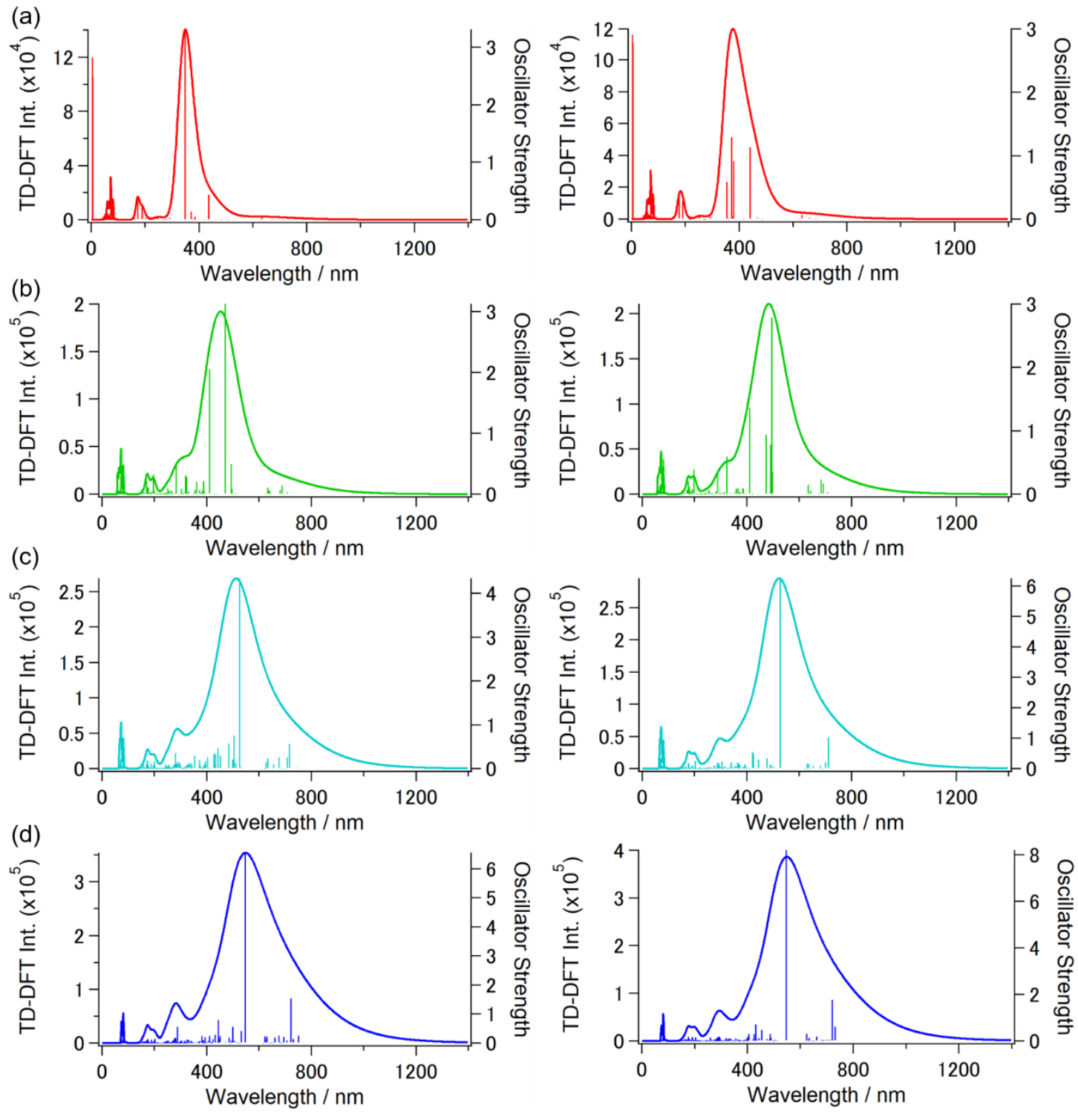

Figure S4. UV-vis spectra of polyiodides in (left column) the gas phase and (right column) water calculated at the CAM-B3LYP/LanL2DZ level. (a) $\mathrm{I}_{5}^{-}$, (b) $\mathrm{I}_{10}{ }^{2-}$, (c) $\mathrm{I}_{15^{3-}}$, and (d) $\mathrm{I}_{20}{ }^{4-}$. These polyiodide systems are prepared by removing $\alpha$-CDs and $\mathrm{Li}^{+} \mathrm{s}$ from the polyiodide $/ \mathrm{Li}^{+} \mathrm{s} / \alpha-\mathrm{CDs}$ system geometrically optimized by QM/MM method (see Figure 6). These UV-vis spectra are numerically calculated by assuming one Gaussian function with a bandwidth of $0.4 \mathrm{eV}$ for each electronic transition. The peak wavelengths for UV-vis spectra of polyiodides in gas phase and water are, respectively, as follows; (a) $348 \mathrm{~nm}$ and $377 \mathrm{~nm}$, (b) $453 \mathrm{~nm}$ and $484 \mathrm{~nm}$, (c) $512 \mathrm{~nm}$ and $523 \mathrm{~nm}$, and (d) $547 \mathrm{~nm}$ and $549 \mathrm{~nm}$. 
(a)
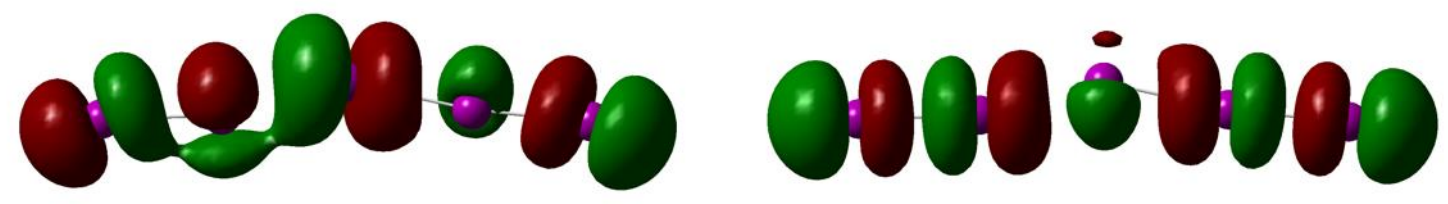

(b)

\subsection{0 .0000000000000000000000}

(c)

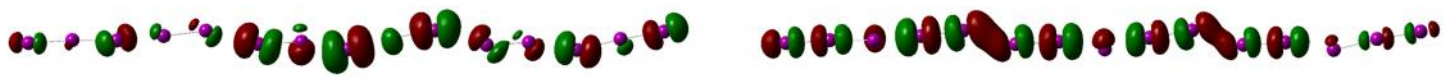

(d)

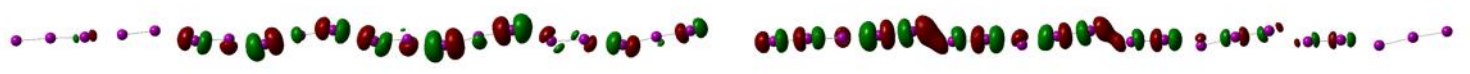

Figure S5. (left column) HOMO and (right column) LUMO molecular orbitals of the polyiodides in the gas phase dominating the electrostatic transitions with the strongest TD-DFT intensities in Figure S4. Each electronic transition occurs between (a) the 18th and 19th states for $\mathrm{I}_{5}^{-}$, (b) the 36th and 37 th states for $\mathrm{I}_{10}{ }^{2-}$, (c) the 54th and 55th states for $\mathrm{I}_{15^{3-}}$, and (d) the 72nd and 73 rd states for $\mathrm{I}_{20}{ }^{4-}$. 
(a)
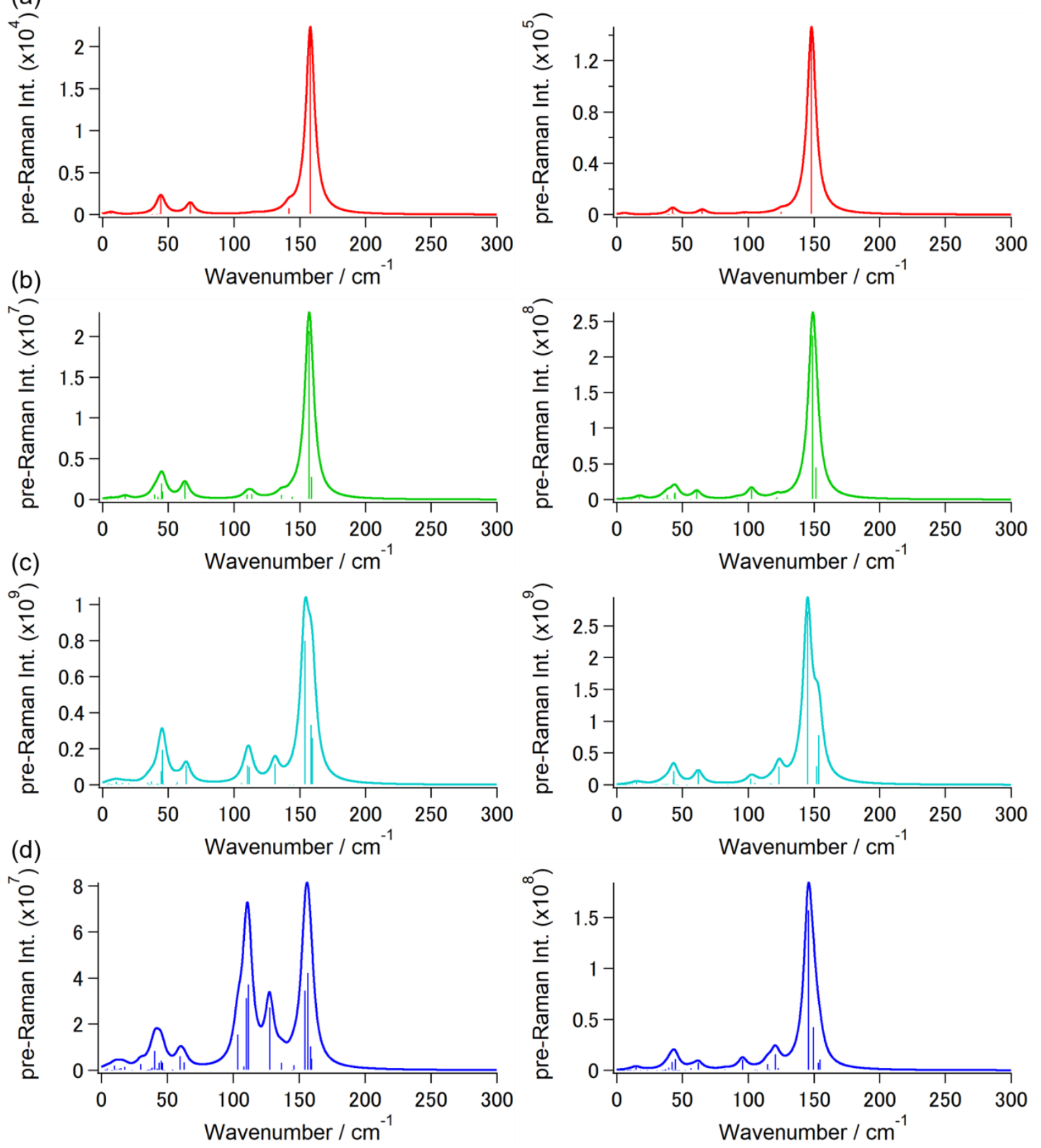

Figure S6. Preresonance Raman spectra of polyiodides in (left column) the gas phase and (right column) water calculated at the CAM-B3LYP/LanL2DZ level. (a) $\mathrm{I}_{5}{ }^{-}$, (b) $\mathrm{I}_{10}{ }^{2-}$, (c) $\mathrm{I}_{15}{ }^{3-}$, and (d) $\mathrm{I}_{20}{ }^{4-}$. These polyiodide systems are prepared by removing $\alpha$-CDs and $\mathrm{Li}^{+} \mathrm{s}$ from the polyiodide $/ \mathrm{Li}^{+} \mathrm{s} / \alpha-\mathrm{CDs}$ system geometrically optimized by QM/MM method (see Figure 6 ). The wavelength of incident electronic field is $514.5 \mathrm{~nm}$. These Raman spectra are numerically calculated by assuming one Gaussian function with a bandwidth of $4 \mathrm{~cm}^{-1}$ for each normal mode. 

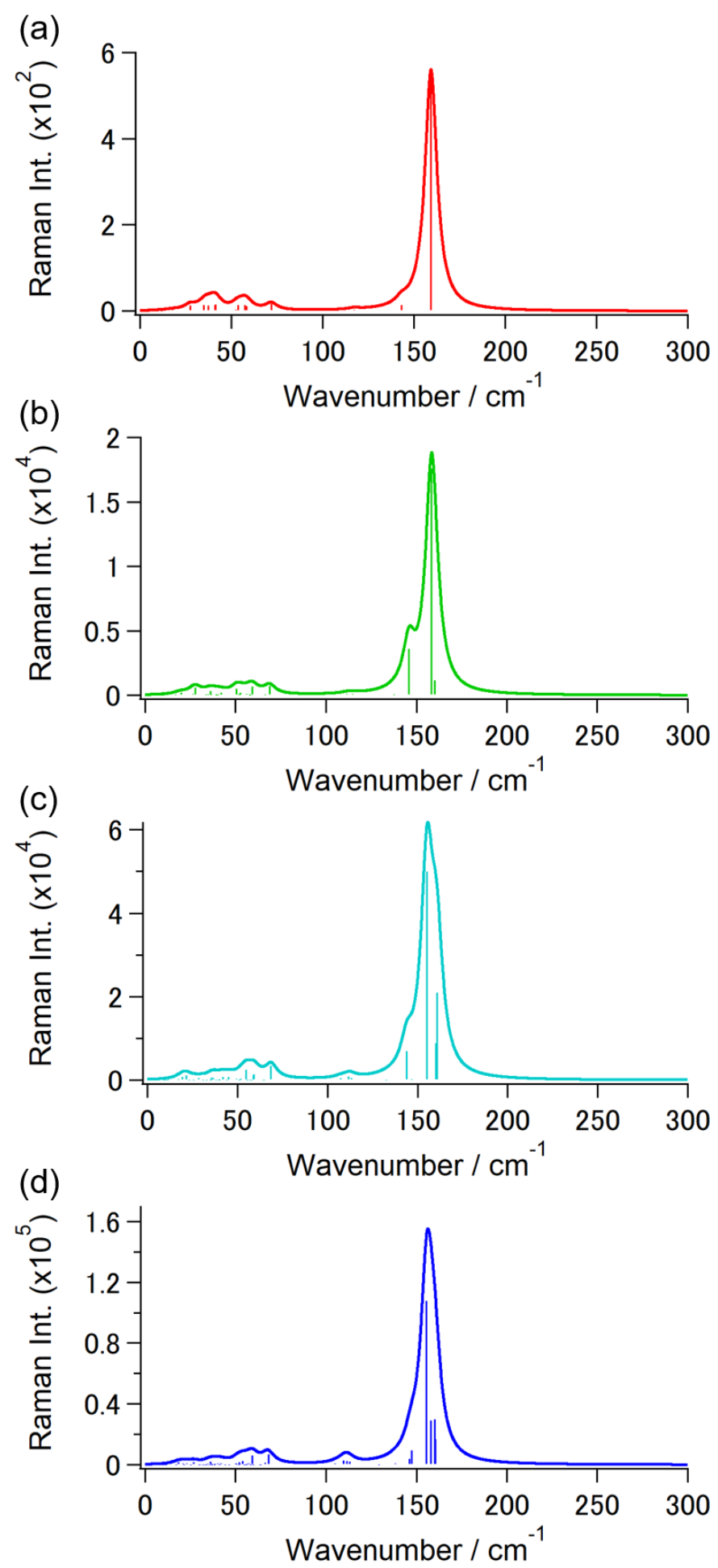

Figure S7. Raman spectra of polyiodide/ $\alpha-\mathrm{CDs} / \mathrm{Li}^{+} \mathrm{s}$ in the gas phase calculated by the QM/MM method. (a) $\mathrm{I}_{5}{ }^{-} / \mathrm{Li}^{+} / 2 \alpha-\mathrm{CD}$, (b) $\mathrm{I}_{10}{ }^{2-} / 2 \mathrm{Li}^{+} / 4 \alpha-\mathrm{CD}$, (c) $\mathrm{I}_{15}{ }^{3-} / 3 \mathrm{Li}^{+} / 6 \alpha-\mathrm{CD}$, and (d) $\mathrm{I}_{20}{ }^{4-} / 4 \mathrm{Li}^{+} / 8 \alpha-\mathrm{CD}$. These Raman spectra are numerically calculated by assuming one Gaussian function with a bandwidth of $4 \mathrm{~cm}^{-1}$ for each normal mode. 